\title{
Velocidad de lectura, prosodia, y resultados de comprensión
}

\author{
Sara Recio-Pineda \\ Universidad de Barcelona \\ srecio@ub.edu
}

\section{Resumen}

El impacto que tradicionalmente ha tenido la teoría de la automaticidad en la explicación del desarrollo lector, junto al auge de la preocupación por que los escolares adquieran una mayor fluidez lectora, ha provocado una proliferación de estudios que han puesto su foco de atención en la velocidad de los lectores, pero que, por lo general, se han olvidado de interpretar qué papel cumple la prosodia. El presente trabajo tiene por objetivo explicar cómo afecta, por un lado, el tiempo que 72 escolares de primaria provinientes de dos escuelas públicas de Cataluña invierten en leer un relato adecuado a su edad y, por el otro, la calidad de su prosodia a los resultados de comprensión. Se utiliza, para ello, un método cuantitativo correlacional. Los resultados permiten concluir que son las estrategias prosódicas (y no la duración de la lectura) las que afectan a la calidad de la comprensión.

Palabras clave: Fluidez, Prosodia, Velocidad, Comprensión, Lectura

\section{Resumo}

O impacto que a teoria da automaticidade tem tradicionalmente na explicação do desenvolvimento do leitor, juntamente com o aumento da preocupação de que os alunos adquiram maior fluência na leitura, causou uma proliferação de estudos que focaram a atenção na velocidade dos leitores, mas que, em geral, se esqueceram de interpretar qual o papel da prosódia. O objetivo deste artigo é explicar como isso afeta, por um lado, o tempo em que 72 alunos do ensino fundamental de duas escolas públicas da Catalunha investem na leitura de uma história apropriada à sua idade e, por outro, na qualidade de sua prosódia aos resultados da compreensão. Um método correlacional quantitativo é usado para isso. Os resultados permitem concluir que são as estratégias prosódicas (e não a duração da leitura) que afetam a qualidade da compreensão.

Palavras chave: Fluência, Prosódia, Velocidade, Compreensão, Leitura 
Velocidad de lectura, prosodia, y resultados de comprensión...

\begin{abstract}
The impact that the theory of automaticity has traditionally had on the explanation of reader development, together with the rise of concern that schoolchildren should acquire greater reading fluency, has caused a proliferation of studies that focus their attention on the speed of the readers. In general, however, these works have forgotten to interpret how reading prosody affects comprehension. The purpose of this paper is to explain how the variables of speed and prosody affect comprehension on a sample of 72 primary school students. A quantitative correlational method is used for this. The results point out that it is the prosody (and not the duration of the reading) what affects the quality of understanding in reading.
\end{abstract}

Keywords: Fluency, Prosody, Speed, Comprehension, Reading

\title{
1 Introducción
}

De acuerdo con publicaciones tan influyentes como el National Reading Panel (NIHD 2000), la fluidez - definida como el equilibrio entre velocidad, precisión y expresividad - es un factor crítico y predictor del nivel de comprensión lectora que se asuma. Pero tal y como indica Calero (2014, p.34), a lo largo de casi un siglo, se "ha implantado en el imaginario colectivo de profesores y alumnos [...] la idea de que una lectura fluida es equiparable a una lectura veloz, [...] asociada a un cronómetro". Y es que, de acuerdo con Kamil et al. (2011), la cantidad de palabras leídas por minuto (rate) ha sido la variable de la fluidez que mayor atención ha recibido por la facilidad de medición que presenta. Como consecuencia, se ha fallado sucesivamente en ahondar en el estudio de otras variables relacionadas con la fluidez.

Tal y como apunta DeStefano (2017), de hecho, son varios los esfuerzos técnicos internacionales que promueven la medición de las CWPM (palabras correctas leídas por minuto) como punto de referencia en la medición de las habilidades lectoras, y este privilegio de la variable velocidad y de la variable precisión ha llegado a afirmarse, incluso, y por separado, como representativa del nivel de comprensión de los escolares. Para Nuttall (1996) y Champeau de López (1993), la velocidad lectora y la comprensión de lectura están estrechamente relacionadas, en efecto, pero la naturaleza precisa del eslabón entre ellos ha sido el enfoque de un debate.

Así, por ejemplo, Walczyk y Griffin-Ross (2007) indicaron que la velocidad puede estar o no relacionada con la comprensión dependiendo de 
la presencia de otras habilidades compensatorias, como releer o hacer inferencias. Y, de hecho, Adlof, Catts y Little (2006) identificaron, en una muestra de 604 alumnos lectores de inglés de distintos cursos de primaria, que los alumnos con puntajes bajos en velocidad mostraban una comprensión dentro de los límites normales de la muestra. Por su parte, Camarillo-Salazar y Silva-Maceda (2016) encontraron que la velocidad de lectura de pseudopalabras solo explicaba variabilidad en los puntajes de comprensión de manera significativa en una fase de lectura inicial, pero que una vez que la muestra de lectores había alcanzado mayor fluidez, la velocidad ya no constituía un indicador de comprensión válido.

En las teorías sobre la descodificación, sin embargo, se entiende que el procesamiento adicional que requiere codificar un texto se hace posible una vez que las palabras pueden ser procesadas con fluidez y de manera automática por el lector, por lo que la automaticidad — que se mide a través de la velocidad y precisión de nombramiento de palabras o frases - debe ser una preocupación de primer orden entre los interesados en el desarrollo de las habilidades lectoras. Parafraseando a Escurra (2003, p.108), se piensa que el lector lento se detiene en paquetes pequeños de información del texto y que, por consiguiente, su memoria de trabajo debe realizar un mayor esfuerzo para poder retener la información, lo que provoca que su proceso de comprensión se vea entorpecido. Esto podría explicar el interés por relacionar la velocidad de reconocimiento, y el nombramiento y la lectura de palabras y frases de forma correcta con la comprensión.

En la línea de lo anterior, Gough y Tumner's (1986) afirman que si el niño no ha desarrollado suficiente automaticidad, su comprensión se verá perjudicada. Y siguiendo esta preocupación, Kaminski y Good (1998) desarrollaron el test Dynamic Indicators of Basic Early Literacy Skills, más comúnmente conocido por su acrónimo: DIBELS. Este test mide la velocidad, la precisión y las pausas de los escolares en el transcurso de un minuto de lectura en voz alta y ha sido usado en las escuelas y estudios como el de Petscher y Kim (2011), entre otros. En esta línea también conviene citar el test GORT (Gray Oral Reading Test, GRAY; ROBINSON, 1967) y el test CBM (Currículum-Based Measures, MARSTON, 1989). Todos estos tests se caracterizan por incluir una fase de medición de cuántas palabras leen los participantes de forma correcta en el primer minuto del ejercicio y por prestar mucha atención a las pausas de los lectores. 
De acuerdo con Dowd y Barlett (2019, p.192), además, los subtest EGRA (Early Grade Reading Assessment, GOVE, 2008) - que incluyen mediciones de la velocidad en el nombramiento de letras, sílabas, palabras sin sentido y palabras familiares y que contienen, entre otros test, una prueba en la que se ofrece a los informantes un tiempo delimitado para leer en voz alta la mayor extensión posible de un pasaje corto y contestar a unas preguntas de comprensión asociadas a la parte que se haya conseguido leer - han sido aplicados en más de 72 países. Y, de nuevo, tal y como advierten estas autoras, este conjunto de test sobrevalora el indicador de la velocidad y el uso del cronómetro. De hecho, en la prueba en la que hay que leer en voz alta tanto fragmento de texto como se pueda en un tiempo delimitado, solo los lectores más rápidos pueden contestar a todas las preguntas de comprensión del test, así que se concluye de forma confusa que la comprensión requiere rapidez.

Jenkins et al. (2003), entre muchos otros (véase, también, LEÓNISLAS; MAY; CHI, 2019), se han dedicado a estudiar la relación predictora de la velocidad y la precisión al leer con las habilidades de comprensión lectora. También investigaciones como las de Fugamalli, Barreyro y Jaichenco (2017) o Yildirim, Rasinski y kata (2017), por ejemplo, han sugerido que la fluidez de lectura de palabras por minuto y la comprensión de textos muestran una correlación positiva significativa. Pero todos estos estudios se han visto fuertemente influenciados por las metodologías y perspectivas de los tests de evaluación de la lectura y las teorías que se han citado con anterioridad.

Pero tal y como indican González-Trujillo et al. (2014, p.118), la teoría de la automaticidad (LABERGE; SAMUEL, 1974), no atiende directamente al papel desempeñado por la prosodia en todo el procesamiento lector.

[...] aprender a leer incluye identificar las unidades nocionales del texto, identificar los grupos de palabras que significan conjuntamente [...]. En el discurso oral, los grupos fónicos vienen dados por la pronunciación del hablante, y en el discurso escrito es el lector el que debe atribuir al texto su organización en grupos fónicos [...] segmentar así en bloques el discurso y, más aún, dar a cada uno una inflexión tonal apropiada (CANTERO, 2002a, p.77-79). 
Sabatini, Wang y O'Reilly (2019), recientemente, han explorado las relaciones que se establecen entre la comprensión lectora y el desempeño en la lectura en voz alta en una muestra de 1713 estudiantes de cuarto curso de primaria utilizando la escala NAEP (DAANE et al., 2005). Los autores de este trabajo concluyen que tanto la precisión en la lectura de palabras como la velocidad de palabras correctas de un fragmento leído en un periodo de un minuto explican los resultados entre los lectores que obtienen menor puntaje de comprensión de la prueba. En este estudio, de nuevo, la comprensión se mide bajo las condiciones de un cronómetro y las conclusiones prestan muy poca atención al papel que desempeña la prosodia.

Pero ya en 2007, Ravid y Mashraki encontraron que las pausas en la lectura de los niños (uno de los factores que más influye a los resultados de velocidad) no estaban tan relacionadas con la comprensión que estos hacían del texto como con sus marcas de entonación. Por consiguiente, la prosodia tendría un mayor efecto que la velocidad para la comprensión, al empaquetar unidades sintácticas y semánticas que se podrían detectar a través de cambios en el tono (pitch) y que ayudaran al lector a comprender.

Por su parte, Tsui, Tong y Fung (2016) pidieron a 10 niños bilingües (cantonés-inglés) que leyeran un pasaje de 12 frases tan bien y expresivamente como pudieran $\mathrm{y}$, paralelamente, les administraron una prueba de comprensión en la que tenían que asociar una imagen a una frase. Calcularon los valores de tono en las inflexiones finales y las duraciones de las pausas y encontraron que solo el tono guardaba alguna relación con los resultados de comprensión (y no las pausas).

[...] Se parte de la idea errónea que considera el incremento en velocidad lectora como la causa, y no como la consecuencia de una lectura fluida; es decir, se asume que si la velocidad se incrementa, la fluidez mejorará también. No obstante, [...] exigir a los alumnos que lean con velocidad supone, en muchos casos, que ambas: su lectura prosódica y su comprensión lectora sufran, se vean disminuidas de un modo significativo (CALERO, 2014, p.39).

Así, podría pensarse que el actual entendimiento de cómo influye la velocidad de lectura en la comprensión es limitado, contradictorio y controvertido. Conviene, todavía, desarrollar un acercamiento al desarrollo lector que cuente con mayor bibliografía empírica, y que sea más sensible a 
las diferencias culturales, linguiísticas e individuales de las muestras de estudio, así como a las condiciones bajo las que se realizan las pruebas de evaluación. Se ha prestado poca atención a cómo afecta la velocidad a la comprensión cuando no hay restricciones de tiempo en la prueba a la que se someten los informantes $\mathrm{y}$, sobre todo, cuando el informante percibe que su foco debe estar puesto en la comprensión. Pero, además, y sobre todo, surge la necesidad de comprender el papel de la prosodia en los puntajes totales de comprensión y de velocidad.

\section{Método}

Con el fin de explorar algunos de los vacíos que ofrece la literatura, este trabajo se propone describir

a) la relación que existe entre el tiempo normal que cada lector joven invierte en leer en voz alta un relato adecuado a su edad y la calidad de su comprensión lectora

b) la relación que existe entre la calidad de la prosodia de cada lector joven y la calidad de su comprensión lectora

c) la relación que existe entre el tiempo normal que cada lector joven invierte en leer en voz alta un relato adecuado a su edad y la calidad de su prosodia

d) el efecto relativo del tiempo que cada lector joven invierte en leer un relato adecuado a su edad, y de la prosodia, conjuntamente, sobre la calidad de su comprensión lectora

Para abordar estos objetivos, se utilizará un método cuantitativo correlacional.

\subsection{Participantes}

La muestra está formada por setenta y dos participantes - 36 niñas y 35 niños-que se encuentran cursando distintos cursos de Educación Primaria (24 participantes de $2^{\circ}, 4^{\circ}$ y $6^{\circ}$ curso de $\mathrm{EP}^{1}$, respectivamente) y que provienen equitativamente de dos escuelas públicas de Cataluña. Se trata de una muestra equilibrada y heterogénea en niveles de lectura, pues

\footnotetext{
${ }^{1}$ En España, estos cursos albergan a los escolares de 7-8 años, 9-10 años y 11-12 años, respectivamente.
} 
contiene, según indicaciones de las maestras, que fueron las que en última instancia seleccionaron a los participantes de acuerdo a un criterio de conveniencia y disponibilidad, a ocho lectores con un nivel alto de lectura, ocho lectores con un nivel medio y ocho lectores con un nivel bajo de lectura de cada curso (cuatro de cada escuela, respectivamente).

Todos los participantes han sido escolarizados desde preescolar en el mismo centro, lo que implica que fueron expuestos de forma temprana a la lengua escrita y a la lengua catalana (que es la lengua en la que están escritos los relatos que leyeron). El muestreo excluye a lectores con dificultades de aprendizaje debidas a algún diagnóstico conocido como dislexia, sordera o TDHA.

\subsection{Instrumentos}

A continuación, se describen los instrumentos que se utilizaron en el proceso de recogida y análisis de los datos: los textos, las plantillas de posibilidades fónicas de los textos, las preguntas de comprensión y las plantillas con los criterios para su evaluación.

\subsubsection{Los textos}

Se seleccionaron y extrajeron, de libros de texto ${ }^{2}$ de Primaria, y de acuerdo a las tres franjas de edad de la muestra, tres relatos auténticos ${ }^{3}$ que tenían una página de extensión aproximada. El texto que leyeron los participantes de $2^{\circ}$ aparecía en un libro de texto destinado a estudiantes de $1^{\circ}$ curso y narra las aventuras de una piedra que quiere ver el mar. El texto que leyeron los participantes de $4^{\circ}$ curso aparecía en un libro de texto destinado a estudiantes de $3^{\circ}$ curso y explica el misterio de un pueblo cuyos habitantes se caracterizan por tener un elevado índice de felicidad. Por último, el texto que leyeron los participantes de $6^{\circ}$ curso, y que también una editorial había seleccionado para una franja de edad anterior a la de los participantes, explica lo que le ocurre a Jaume, un hombre muy peculiar por

${ }^{2}$ En Cataluña, la lengua vehicular de las escuelas es el catalán y esto significa que los libros de texto están editados en esta lengua. También es el catalán la lengua en la que los participantes del estudio iniciaron su aprendizaje lector formal y participaron en la investigación. 
el hecho de ser transparente, tanto en un sentido literal, como en un sentido metafórico, cuando llega a su pueblo un malvado dictador al que detesta.

Los relatos fueron seleccionados por su carácter narrativo lineal (sin flashbacks), sus características de adecuación a la edad (de hecho, fueron publicados para ser leídos en un curso escolar anterior al de los participantes), su extensión de una página aproximada, y por estar redactados con un estilo discursivo rico, que se caracterizara por la presencia frases largas que pusieran a prueba la prosodia del lector. Se presentaron en una hoja plastificada que mantenía el formato de letra de la editorial y eliminaba las imágenes para asegurar que la evaluación de la comprensión de los participantes tomara solo en cuenta la información escrita.

\subsubsection{Las plantillas de posibilidades fónicas de los textos}

Estas plantillas describen de cuántas maneras distintas se pueden agrupar prosódicamente los enunciados e ideas de los distintos textos sin que se rompan las estructuras de solidaridad sintáctica, ni se incurra en estructuras incoherentes con los signos de puntuación (véase NAVARROTOMÁS, 1944; QUILIS, 1981 o MARTÍN-BUTRAGUEÑO, 2003). Sirven como punto de referencia para comparar la entonación prelingüística $^{4}$ de los diversos participantes con un modelo común de referencia.

La plantilla se elaboró con la colaboración de tres investigadores expertos en prosodia. Primero, el equipó identificó, de forma individual, los grupos fónicos (cfr. CANTERO, 2002b) más extensos posibles en cada enunciado del texto y, después, identificó las combinaciones de grupos fónicos menores que podría haber dentro de un mismo enunciado. Las tres impresiones individuales se cruzaron, analizaron y discutieron para crear

\footnotetext{
${ }^{4}$ La forma de integrar y delimitar el habla (o los enunciados leídos) dentro de bloques de sonidos que están fónicamente jerarquizados pertenece al ámbito de análisis del nivel prelingüístico de la entonación. En este nivel de análisis, la unidad mayor de sonidos organizados se denomina grupo fónico - que, de acuerdo Mateo (2010, p.51) se define como la "sucesión de tonos (...) organizados en torno a una inflexión final" o núcleo entonativo-o dicho de otro modo: como el conjunto de sonidos agrupados alrededor de un acento de frase, que puede identificarse mediante una inflexión final.
} 
una plantilla final. Tras analizar cómo agrupaban prosódicamente las unidades fónicas del texto los participantes, las plantillas volvieron a revisarse para valorar si había alguna combinación realizada por la muestra que no se hubiera tenido en cuenta y que siguiera con los criterios de validez para considerarlo un grupo fónico: solidaridad entonativa, sintáctica y de puntuación.

\subsubsection{Las preguntas de comprensión y las plantillas para su evaluación}

De cada texto se formularon ocho preguntas abiertas de comprensión que se dirigían a la identificación del argumento, el tema, los personajes, el problema, la acción, la resolución o la moraleja. Se trata, en definitiva, de preguntas que obligaban a poseer una representación global del significado del texto para ser respondidas y que no podían contestarse con una sola palabra.

Para evaluar las respuestas asociadas a cada pregunta se establecieron cuáles eran las claves temáticas mínimas (que no palabras exactas) que debían aparecer en las respuestas, atendiendo a criterios de coherencia y de adecuación con los índices del texto. Debe entenderse que las respuestas que contenían todas las claves temáticas indicadas en esta plantilla se evaluaron como totalmente satisfactorias. El resto de casos se evaluaron como parcialmente satisfactorios o como respuestas nulas.

\subsection{Procedimiento}

Tras conseguir el consentimiento informado de los padres, las maestras de cada curso y escuela seleccionaron a los 72 lectores noveles que formarían parte de la investigación. El objetivo último de la selección era que la muestra fuera lo más amplia y heterogénea posible y que contuviera el mismo número de participantes en cada escuela, en cada curso y en cada nivel de competencia lectora.

En una sala especialmente habilitada para la recogida de datos, se grabó a cada uno de los participantes leyendo en voz alta el relato que se había seleccionado para su franja de edad. En la instrucción de la "actividad", los participantes fueron informados de que debían leer para comprender los aspectos globales de la trama y comentarlos después (de qué trata, quiénes y cómo son los personajes, cómo acaba, etc.). Se les informó de que no importaba la velocidad ni la pronunciación de su lectura 
y que el foco de atención, en cualquier caso, debía estar puesto en comprender lo mejor posible el relato, ya que después pasarían a comentar con la investigadora, de forma oral, su impresión sobre los aspectos más importantes de la historia (esto es: a contestar a las preguntas de comprensión en una dinámica dialógica). Luego, se les ofreció algunos ejemplos del tipo de cuestiones que comentarían con la investigadora: de qué trata el relato, qué pasa en él, quiénes son los personajes, cómo acaba...

Se les indicó, en pos de alcanzar la mejor comprensión posible del texto, que si perdían el hilo de lo que leían, volvieran atrás y releyeran lo que necesitaran. También se les permitió que, previo a la lectura, realizaran unas primeras hipótesis acerca de lo que creían que iba a tratar el relato para centrar su atención. Esto último se hizo a través de las pistas que ofrecía el título de cada relato.

Se anotó el tiempo (en segundos) que cada uno de los informantes invirtió en leer el relato; se analizó la prosodia de los lectores valorado cuántas de sus marcas prosódicas seleccionaban grupos fónicos previstos por la plantilla de posibilidades fónicas y cuántas no, y, por último, se evaluaron sus respuestas en la entrevista de comprensión.

En un intento por hacer que los participantes se sintieran cómodos en el transcurso de la prueba y que no percibieran que la velocidad de su lectura iba a ser importante, se optó por medir el tiempo que cada uno de ellos invirtiera en la lectura anotando la hora de inicio y de finalización en una libreta y sin la presencia de un cronómetro a la vista.

El primer paso en el análisis de la prosodia leída de los escolares fue describir, tras la escucha repetida de las grabaciones, el modo en el que cada participante organizó fónicamente el texto; es decir, describir en qué lugares del texto los participantes realizaban inflexiones o cesuras. Para hacer esto, se utilizó una libreta que contenía tantas copias del texto como participantes había (24 para cada texto) y se anotó, sobre las líneas textuales, el lugar donde recaían las inflexiones y cesuras de los participantes; esto es: la transcripción de las marcas prosódicas de cada participante. En caso de reparaciones en la lectura, se valoraron solo las marcas prosódicas de la última versión leída.

El análisis de la prosodia se hizo comparando las marcas prosódicas que realizaba cada uno de los informantes al leer con las marcas prosódicas que preveía la plantilla de posibilidades fónicas del texto. Se anotó el número de grupos fónicos que los participantes codificaban tal y 
como preveía la plantilla de posibilidades fónicas; es decir: sin ninguna otra marca prosódica que interrumpiera el grupo fónico previsto por la plantilla de por medio. Y se denominó a esta variable: número de Aciertos Prosódicos o AP.

Después, estos datos se convirtieron en un índice que permitiera comparar y contrastar la cantidad de AP de toda la muestra por igual, independientemente de si habían leído un texto u otro, ya que debe tenerse en cuenta que cada texto tiene una plantilla de posibilidades fónicas distintas $\mathrm{y}$, por lo tanto, prevé un número de AP máximo y mínimo distinto $^{5}$.

Este índice recibió el nombre de IAP (Índice de Aciertos Prosódicos). El lector puede consultar cómo calcularlo en Recio-Pineda (2017, p.272-273). Para los intereses de este trabajo, conviene indicar que el valor de IAP siempre oscila entre 0 y 1, porque la plantilla prevé un número limitado máximo de Aciertos Prosódicos. En este caso, el valor 1 describiría al participante que ha leído todo el texto siguiendo las previsiones de grupos fónicos de la plantilla de posibilidades fónicas y el valor 0 describiría al participante que ha ido introduciendo pausas, inflexiones o cesuras (es decir, ha interrumpido) en todos los grupos fónicos previstos por la plantilla.

Las respuestas a las preguntas abiertas de comprensión se evaluaron teniendo en cuenta a si hacían referencia a todas, algunas o ninguna de las claves temáticas mínimas que quedaron previstas en la plantilla de respuestas ${ }^{6}$.

A continuación, la tabla 1 resume los distintos parámetros que se han utilizado para medir las estrategias prosódicas, la calidad de la comprensión lectora y el tiempo invertido en la lectura de cada participante:

\footnotetext{
${ }^{5}$ Las plantillas de posibilidades fónicas distinguen entre grupos fónicos que son opcionales y grupos fónicos que son obligatorios (porque marcan el final de un enunciado, por ejemplo). El número mínimo de AP que debe contabilizarse corresponde al número de grupos fónicos obligatorios.

${ }^{6}$ Las preguntas se contestaron en una entrevista oral para facilitar que los informantes pudieran reformular o ir ampliando sus respuestas mientras hablaban con la investigadora.
} 
Velocidad de lectura, prosodia, y resultados de comprensión...

Tabla 1 Parámetros de medición del estudio

\begin{tabular}{|l|l|l}
\hline \multicolumn{3}{|c}{ Parámetros de medición } \\
\hline $\begin{array}{l}\text { De las estrategias } \\
\text { prosódicas }\end{array}$ & $\begin{array}{l}\text { De la calidad de la } \\
\text { compresión lectora }\end{array}$ & $\begin{array}{l}\text { Del tiempo invertido } \\
\text { en la lectura }\end{array}$ \\
\hline $\begin{array}{l}\text { Índice de Aciertos } \\
\begin{array}{l}\text { Prosódicos (IAP): Este } \\
\text { índice valora del 0 } \\
\text { (ninguno) al 1 (todos) la }\end{array}\end{array}$ & $\begin{array}{l}\text { Número de respuestas } \\
\text { totalmente satisfactorias o } \\
\text { correctas registradas en la } \\
\text { entrevista }\end{array}$ & Segundos \\
cantidad de grupos fónicos \\
$\begin{array}{l}\text { adecuados (idénticos a los } \\
\text { previstos en la plantilla) que } \\
\text { cada uno de los }\end{array}$ & & \\
\hline
\end{tabular}

Fuente: La autora

Al finalizar la recogida de datos, y para el propósito del análisis, se aplicó un cálculo de correlaciones entre las distintas variables con ayuda del programario SPSS. Primero, se realizó un análisis entre pares de variables y, después, se realizó un análisis de la regresión múltiple que permitiera clarificar mejor la relación entre ellas.

\section{Resultados}

A continuación, se presentan los resultados que se obtuvieron del análisis correlacional bivariado. Primero, se muestra la estadística correlacional entre la variable del tiempo invertido en la lectura por cada participante (medida en segundos) y los resultados de comprensión (o cantidad de respuestas correctas). Después, se muestran los resultados de correlacionar la variable del tiempo invertido en la lectura por cada participante (segundos) y la calidad de su prosodia (medida a través de la variable IAP). Luego, se muestra la correlación entre la calidad de la prosodia de los informantes (IAP) y sus resultados en la prueba de comprensión.

Para finalizar, se muestran los resultados de analizar el efecto conjunto del tiempo que cada lector joven invierte en leer el relato y de la calidad de su prosodia sobre los resultados de comprensión. 


\subsection{Relación entre el tiempo de lectura y los resultados de comprensión}

En el Gráfico 1 se muestra la relación entre las variables segundos y respuestas correctas.

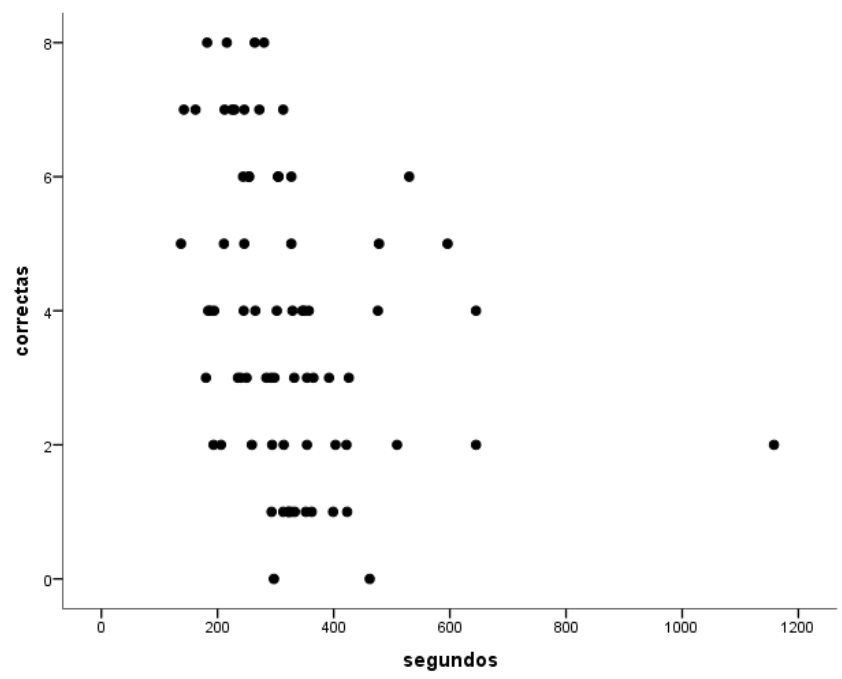

Gráfico 1. Relación entre el tiempo de lectura y los resultados de comprensión Fuente: La autora. Producido con SPSS.

Debido a que no se cumplen las condiciones para aplicar una correlación de Pearson (principio de homocedasticidad), se optó por emplear la correlación de Spearman. La correlación entre ambas variables (tiempo invertido en la lectura y resultados de comprensión) es negativa, moderada y significativa $\left(r_{s}=-0.423 ; p<0.001\right)$. Es decir, a medida que aumentan los segundos, disminuye el número de respuestas correctas.

\subsection{Relación entre la calidad de la prosodia y los resultados de comprensión}

La correlación de Spearman entre el índice IAP y el número de respuestas correctas (véase Gráfico 2) es positiva, moderada y significativa $\left(r_{s}=-0.372 ; p<0.001\right)$. Es decir, a medida que aumenta el puntaje en IAP, aumenta también el puntaje de comprensión. 
Velocidad de lectura, prosodia, y resultados de comprensión...

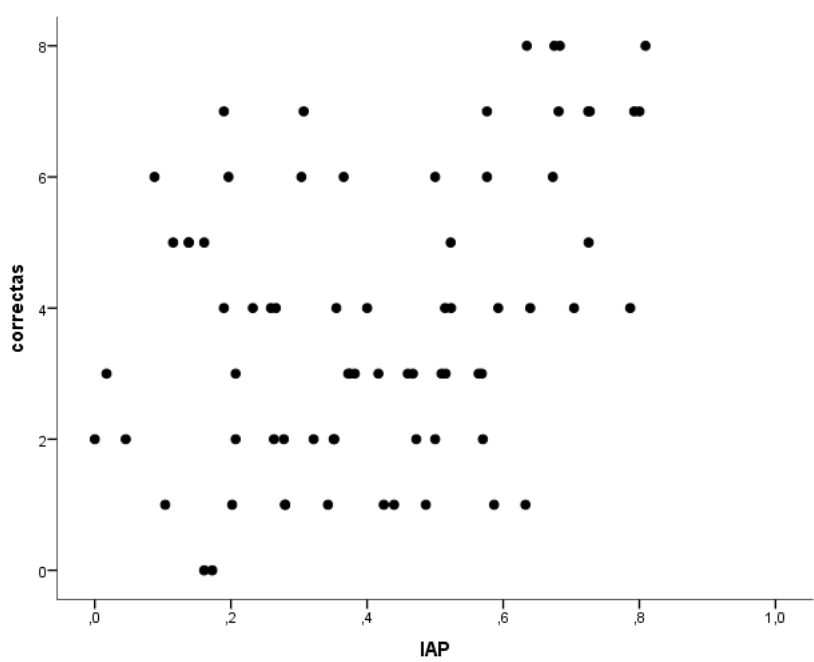

Gráfico 2. Relación entre el tiempo de lectura y el Índice de Aciertos Prosódicos Fuente: La autora. Producido con SPSS.

\subsection{Relación entre el tiempo de lectura y la calidad de la prosodia}

En el Gráfico 3, se muestra la relación entre las variables segundos e IAP. Tal y como puede percibirse, la dispersión no es homogénea para todos los valores de IAP, así que se optó por emplear, de nuevo, la correlación de Spearman. La correlación de Spearman entre el tiempo invertido en la lectura y el Índice de Aciertos Prosódicos es negativa, moderada y significativa $\left(r_{s}=-0.523 ; p<0.001\right)$. Es decir, a medida que aumentan los segundos, disminuye el valor del índice IAP y a la inversa: cuanto menos tarda el lector en leer, es posible, también, que su puntaje en el índice IAP sea más alto. 


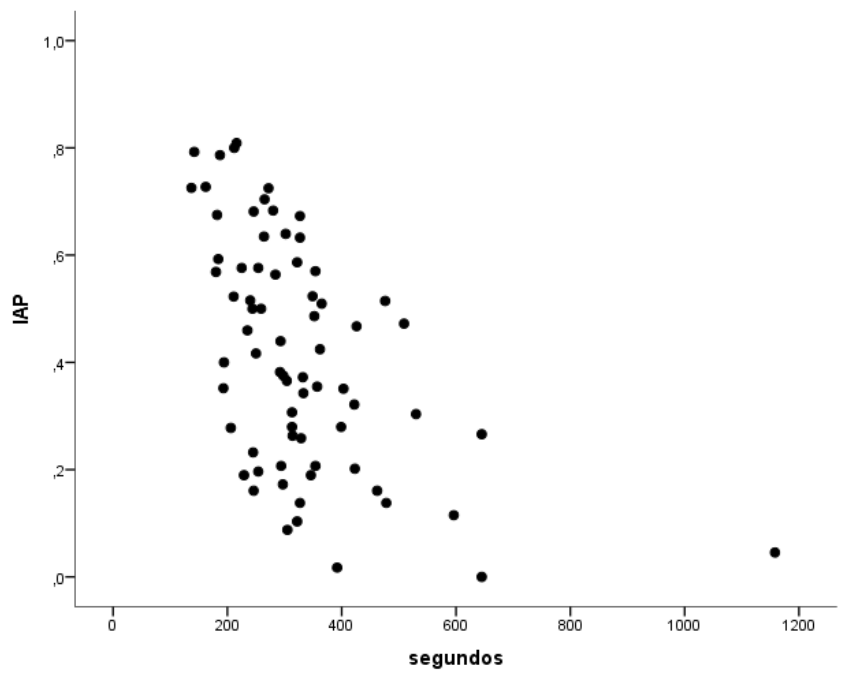

Gráfico 3. Relación entre el tiempo de lectura y el Índice de Aciertos Prosódicos Fuente: La autora. Producido con SPSS.

\subsection{Efecto conjunto del tiempo invertido en la lectura y de la prosodia sobre los resultados de comprensión}

Los resultados anteriores muestran correlaciones moderadas entre el tiempo invertido en la lectura y la cantidad de respuestas correctas que se contestan, por un lado, y entre el tiempo invertido en la lectura y la calidad de la prosodia leída, por el otro. También la correlación entre prosodia y comprensión es significativa y moderada.

Con el fin de clarificar mejor el efecto que tienen tanto el tiempo de lectura como la prosodia sobre los resultados de comprensión, y de ahondar en la relación entre las tres variables, se procede, a continuación, a realizar un análisis conjunto a través de un modelo de regresión lineal múltiple.

Antes, empero, conviene notar que un análisis de los supuestos del modelo de regresión lineal múltiple indica que hay problemas con el cumplimiento del supuesto de normalidad de los residuos. Ante esto, se opta por realizar los modelos regresión mediante simulación de muestreo (Bootstrap) extrayendo 1000 muestras. La tabla 2 muestra un resumen de este modelo. Debe notarse que se ha optado por convertir el IAP en un 
Velocidad de lectura, prosodia, y resultados de comprensión...

porcentaje (IAP_PORC) que facilite la interpretación y comprensión de los datos.

Tabla 2. Resumen del modelo de regresión múltiple entre las tres variables mediante una simulación de muestreo

\begin{tabular}{cc|c|c|c|c}
\hline \multicolumn{7}{c}{ Resumen del modelo $^{\mathrm{b}}$} \\
\hline Modelo & $\mathrm{R}$ & R cuadrado & $\begin{array}{c}\text { R cuadrado } \\
\text { ajustado }\end{array}$ & $\begin{array}{c}\text { Error estándar } \\
\text { de la } \\
\text { estimación }\end{array}$ & Durbin-Watson \\
\hline 1 &, $420^{\mathrm{a}}$ &, 177 &, 153 & 2,032 & 1,761 \\
\hline
\end{tabular}

a. Predictores: (Constante), IAP_PORC, segundos

b. Variable dependiente: correctas

Fuente: La autora. Producido con SPSS.

Tal y como se puede observar en la Tabla 2, el modelo de regresión múltiple mediante simulación de muestreo indica que las variables prosodia y tiempo explican un $15,3 \%$ de la varianza de la variable comprensión. A continuación, se muestran los coeficientes de este modelo en la Tabla 3.

Tabla 3. Simulación de muestreo para los coeficientes de regresión

Simulación de muestreo para coeficientes

\begin{tabular}{llc|c|c|c|c|c}
\hline & & \multicolumn{4}{c}{ Simulación de muestreo } \\
\cline { 3 - 8 } & & & & \multicolumn{3}{c}{$\begin{array}{c}\text { Intervalo de } \\
\text { confianza al 95\% }\end{array}$} \\
\cline { 3 - 8 } & Modelo & B & Sesgo & $\begin{array}{c}\text { Error } \\
\text { estándar }\end{array}$ & $\begin{array}{c}\text { Sig. } \\
\text { (bilateral) }\end{array}$ & Inferior & Superior \\
\hline 1 & (Constante) & 3,043 &, 153 & 1,147 &, 007 &, 966 & 5,503 \\
\cline { 2 - 8 } & segundos &,- 002 &, 000 &, 002 &, 250 &,- 008 &, 001 \\
\cline { 2 - 8 } & IAP_PORC &, 035 &,- 001 &, 014 &, 011 &, 007 &, 061 \\
\hline
\end{tabular}

Variable dependiente: correctas

a. Los resultados de la simulación de muestreo se basan en 1000 muestras de simulación de muestreo

Fuente: La autora. Producido con SPSS.

Mirando los coeficientes de la Tabla 3, se observa, sin embargo, que el efecto de la variable segundos sobre la variable comprensión no es 
significativo $\left(\mathrm{B}_{1}=-0.002, \mathrm{p}=0.25\right.$, IC: $\left.[-0.008 ; 0.001]\right)$. En cambio, el efecto de la prosodia sobre la comprensión sí lo es $\left(\mathrm{B}_{2}=0.035, \mathrm{p}=0.011\right.$, IC: [0.007; 0.061]). Puesto que estos resultados indican que la variable segundos no es significativa, se procede a eliminarla para obtener el modelo de regresión definitivo. La Tabla 4 muestra un resumen de este último modelo bootstrap, cuando se elimina del análisis la variable comprensión.

Tabla 4. Resumen del modelo de regresión lineal (sin segundos) mediante una simulación de muestreo

\begin{tabular}{cc|c|c|c|c}
\hline \multicolumn{7}{c}{ Resumen del modelo $^{\mathrm{b}}$} \\
\hline Modelo & $\mathrm{R}$ & $\mathrm{R}$ cuadrado & $\begin{array}{c}\text { R cuadrado } \\
\text { ajustado }\end{array}$ & $\begin{array}{c}\text { Error estándar de } \\
\text { la estimación }\end{array}$ & $\begin{array}{c}\text { Durbin- } \\
\text { Watson }\end{array}$ \\
\hline 1 &, $405^{\mathrm{a}}$ &, 164 &, 152 & 2,033 & 1,766 \\
\hline
\end{tabular}

a. Predictores: (Constante), IAP_PORC

b. Variable dependiente: correctas

Fuente: La autora. Producido con SPSS.

Tal y como se puede apreciar en la Tabla 4, el modelo de regresión simple indica que la variable prosodia explica un $15,2 \%$ de la varianza de la variable comprensión. A continuación, la Tabla 5 muestra los coeficientes de la regresión simple (solo con los efectos de la prosodia sobre la comprensión).

Tabla 5. Simulación de muestreo para el coeficiente de IAP

\begin{tabular}{|c|c|c|c|c|c|c|c|}
\hline \multicolumn{8}{|c|}{ Simulación de muestreo para Coeficientes } \\
\hline \multirow{3}{*}{\multicolumn{2}{|c|}{ Modelo }} & \multirow[b]{3}{*}{ B } & \multicolumn{5}{|c|}{$\begin{array}{l}\text { Simulación de muestreo } \\
{ }^{\mathrm{a}}\end{array}$} \\
\hline & & & \multirow{3}{*}{$\begin{array}{r}\text { Sesgo } \\
-, 003\end{array}$} & \multirow{3}{*}{$\begin{array}{c}\begin{array}{c}\text { Error } \\
\text { estándar }\end{array} \\
, 553\end{array}$} & \multirow{3}{*}{$\begin{array}{c}\begin{array}{c}\text { Sig. } \\
\text { (bilateral) }\end{array} \\
001\end{array}$} & \multicolumn{2}{|c|}{$\begin{array}{c}\text { Intervalo de } \\
\text { confianza al } 95 \%\end{array}$} \\
\hline & & & & & & Inferior & Superior \\
\hline 1 & (Constante) & 2,099 & & & & 1,022 & 3,183 \\
\hline & IAP_PORC &, 042 &, 000 & 012 & 001 & ,016 &, 063 \\
\hline
\end{tabular}

Variable dependiente: correctas

a. Los resultados de la simulación de muestreo se basan en 1000 muestras de simulación de muestreo

Fuente: La autora. Producido con SPSS. 
Velocidad de lectura, prosodia, y resultados de comprensión...

Como se puede observar en la Tabla 5 , el efecto de la prosodia sobre la comprensión es significativo $\left(\mathrm{B}_{1}=0.042, \mathrm{p}=0.001, \mathrm{IC}\right.$ : [0.016; 0.063]).

\section{Discusión}

En un inicio, y gracias al análisis correlacional bivariado, se ha observado que, en la muestra y en las condiciones del presente estudio, los resultados de comprensión pueden estar afectados o influidos tanto por el tiempo que cada participante ha invertido en leer el texto como por la calidad de su prosodia. Las correlaciones entre el tiempo de lectura y la comprensión, por un lado; entre el tiempo de lectura y la prosodia, por el otro; y también entre la prosodia y la comprensión son significativas y moderadas, aunque la más acentuada de ellas es la que se establece entre las variables de tiempo invertido en la lectura y prosodia. Esto hace pensar en una relación de dependencia.

Al intentar explicar los resultados de comprensión desde el efecto de la prosodia y del tiempo que cada participante invierte en leer conjuntamente; esto es, suponiendo que las dos variables independientes pueden tener un estatus asimétrico o de dependencia, se observa que la variable que mejor puede explicar la comprensión es la prosodia (y no el tiempo de lectura) y que aquello que explica la prosodia sobre los resultados de comprensión es suficiente para descartar la explicación que da el tiempo. El efecto de la prosodia, en definitiva, es mayor y más redundante.

Tal y como se ha podido observar en la Tabla 2, de hecho, el modelo de regresión múltiple mediante simulación de muestreo indica que las variables prosodia y tiempo explican un $15,3 \%$ de la varianza de la variable comprensión. Pero casi la totalidad de esta varianza $(15,2 \%)$, como se ha podido observar en la Tabla 4, viene explicada por la prosodia.

Dicho de otro modo, el tiempo que los lectores noveles invierten en leer tiene un reflejo en la calidad de sus estrategias prosódicas (IMPnp/seg, IAP/seg) y en su comprensión. Pero son las estrategias prosódicas (y no la duración de la lectura) las que afectan a la calidad de la comprensión. Se debe tener en cuenta, en efecto, que la velocidad normal de lectura puede ser directamente proporcional al Número de Marcas Prosódicas que codifican los participantes, pero el hecho de que los informantes incurran 
en menos o más Marcas Prosódicas no implica que codifiquen más o menos estrategias prosódicas de calidad. De ahí, entonces, que convenga fijarse en la calidad de la prosodia.

La anterior discusión pone de relieve la posibilidad de empezar a deducir, desde un modelo explicativo que debe seguir analizándose en nuevos contextos y muestras, que la duración en tiempo de la lectura no es la clave para la comprensión y sí lo es, en cambio, la calidad de las estrategias prosódicas que se aplican (también de ellas depende la buena aplicación de las estrategias interpretativas y no del tiempo). Esta hipótesis debe acabar de corroborarse en futuros trabajos.

\section{Conclusiones}

De todo el análisis anterior y retomando los objetivos de la presente investigación, se puede observar, en definitiva, que la relación que existe entre el tiempo normal que cada lector joven invierte en leer en voz alta un relato adecuado a su edad y la calidad de su comprensión lectora es moderada pero significativa. Por general, entonces, cuanto más tiempo invierte el lector en leer el relato, menor es el puntaje que obtiene en la prueba de comprensión.

Por otro lado, se averigua que hay una relación positiva, moderada y significativa entre la calidad de la prosodia y los resultados de comprensión. Esto es, que la calidad de la prosodia afecta también a los resultados de comprensión.

Los datos muestran, además, que una mayor necesidad de tiempo para codificar el texto en voz alta viene a menudo explicada por una prosodia deficiente. Esto es, que la correlación entre el tiempo de lectura y la calidad de la prosodia es negativa, moderada y significativa.

Por último, se concluye que la relación que se establece entre el tiempo invertido en la lectura y la calidad de la prosodia del lector es una relación de dependencia. Aquello que explica el tiempo invertido en la lectura sobre la comprensión es consecuencia de lo que puede explicar la prosodia. Y es, por tanto, la prosodia (y no el tiempo) lo que afecta de forma significativa y directa los puntajes obtenidos en comprensión. 
Velocidad de lectura, prosodia, y resultados de comprensión...

\section{Referencias bibliográficas}

Adlof, Suzanne M.; CATTS, Hugh W.; LitTle, Todd. D. Should the simple view of reading include a fluency component? Reading and Writing, v. 19, n. 9, p. 933-958, 2006.

CALERO, Andrés. Fluidez Lectora y Evaluación Formativa. Investigaciones sobre Lectura, n. 1, p. 33-48, 2014. Disponível em: <http://www.redalyc.org/pdf/4462/446243919002.pdf >. Accesso em: 30 ag. 2019.

CAMARILlo-Salazar, Blanca Flor; Silva-MacedA, Gabriela. Relación entre velocidad de lectura y comprensión lectora en alumnos de primaria. Memorias del 8vo Congreso Internacional de Educación, p. 45-59. Cd. Obregón, Sonora: ITESO, 2016.

CANTERO, Francisco J. Oír para leer: la formación del mediador fónico en la lectura. En: MENDOZA, Antonio (Coord.). La seducción de la lectura en edades tempranas. Madrid: Ministerio de Educación, Colección Aulas de Verano, 2002a. p. 75-100.

Teoría y análisis de la entonación. Barcelona: Edicions de la Universitat de Barcelona, 2002b.

Champeau DE LóPEZ, Cheryl. Developing reading speed. Journal of Reading, v. 31, n. 1, p. 50-51, 1993.

DeStefano, Joe. Setting and Using Benchmarks for Reading Performance. Research Triangle Park, NC: RTI International, 2017.

DowD, Amy J.; BARLETT, Lesley. The Need for Speed: Interrogating the Dominance of Oral Reading Fluency in International Reading Efforts. Comparative Education Review, v. 63, n. 2, p. 189-212, 2019.

Gove, Amber. Early Grade Reading Assessment toolkit. World Bank and RTI International, Washington, DC, 2008.

ESCURRA, Miguel. Comprensión de lectura y velocidad lectora en alumnos de sexto grado de primaria de centros educativos estatales y no estatales de Lima. Persona, n. 6, p. 99-134, 2003. Disponível em: <https://www.redalyc.org/pdf/1471/147118110006.pdf>. Accesso em 30 ag. 2019. 
Fugamalli, Julieta Carolina; BARREYro, Juan Pablo; JAICHENCO, Virginia. Niveles de fluidez lectora y comprensión de textos. Traslaciones. Revista Latinoamericana de Lectura y Escritura, v. 4, n. 8, p. 163-186, 2017.

Disponível em: <http://revistas.uncu.edu.ar/ojs/index.php/traslaciones/article/view/1063> Acceso em 7 ag. 2019.

GonzÁlez-Trujillo, M. Carmen et al. Scale of reading fluency in Spanish: measuring the components of fluency/Escala de fluidez lectora en español: midiendo los componentes de la fluidez. Estudios de Psicología, v. 35, n. 1, p. 104-136, 2014.

Gough, Philip B.; Tumner, William E. Decoding, reading, and reading disability. Remedial and Special Education, v. 7, n. 1, p. 6-10, 1986.

Gray, William. S.; RobInson, Helen. M. Gray Oral Reading Test. Indianapolis. In: BOBB-MERRILL. Gray Oral Reading Test, 1967.

JENKINS, Joseph R., et al. Accuracy and Fluency in List and Context Reading of Skilled and RD Groups: Absolute and Relative Performance Levels. Learning Disabilities Research and Practice, v. 18, n. 4, p. 237245, 2003.

KAMIL, Michael L. et. al. (Eds.) Handbook of reading research, Volume IV. New York City, NY: Routledge, 2011.

KAMINSKI, Ruth A.; GOOD, Roland H. Assessing early literacy skills in a Problem-Solving model: Dynamic Indicators of Basic Early Literacy Skills. En: SHINN, Mark R. (Ed.). The Guilford school practitioner series. Advanced applications of Curriculum-Based Measurement. New York, NY, US: The Guilford Press, 1998, p. 113-142.

LABERGE, David; SAMuEL, S. Jay. Toward a theory of automatic information processing in reading. Cognitive Psychology, v. 6, n. 2, p. 293 323, 1974.

LEÓN-ISLAS, Eugenio Elías; MAY, Marisol; CHI, Jorge Antonio. Comprensión lectora y medición de fluidez en universitarios de origen maya. CPU-e, Revista de Investigación Educativa, v. 1, n. 28, p. 152-182, jan. 2019. Disponível em: 〈http://cpue.uv.mx/index.php/cpue/article/view/2603/4487>. Accesso em 23 jul. 2019. 
Velocidad de lectura, prosodia, y resultados de comprensión...

MARSTON, Douglas B. A curriculum-based measurement approach to assessing academic performance: What it is and why do it. En: SHINN, Mark R. (Ed.). The Guilford school practitioner series. Curriculum-based measurement: Assessing special children. New York, NY, US: Guilford Press, 1989, p. 18-78.

MARTín-ButragueÑo, Pedro. Entre la prosodia y la sintaxis: variación melódica en el estilo de lectura. En: Moreno, Francisco et al. (Coord.). Homenaje a Humberto López Morales. Trabajos de sociolingüística. Madrid: Arco-Libros, 2003.

MATEO, Miguel. Protocolo para la extracción de datos tonales y curva estándar en análisis melódico del habla (AMH). PHONICA, v. 6, p. 49-90, 2010 .

Disponível em: <http://www.publicacions.ub.edu/revistes/phonica6/documentos/702.pdf $>$. Acceso em 30 ag. 2019.

DAANE, Mary C. et al. Fourth-grade students reading aloud: NAEP 2002 Special Study of Oral Reading. (NCES 2006-469). Washington, D.C.: U.S. Department of Education, Institute of Education Sciences, National Center for Education Statistics, 2005

NATIONAL READING PANEL. Report of the subgroups: National reading panel. Washington, DC: National Institute of Child Health and Development, 2000.

NAVARro TOMÁs, Tomás. Manual de entonación española. New York: Hispanic Society. Madrid: Guadarrama, 1944.

NuTTALL, Christine. Teaching reading skills in a foreign language. Oxford: Heinemann English Language Teaching, 1996.

PETSCHER, Yaakov; KIM, Young-Suk. The Utility and Accuracy of Oral Reading Fluency Score Types in Predicting Reading Comprehension. Journal of School Psychology, v. 49, n. 1, p. 107-29, 2011.

QUILIS, Antonio. Fonética acústica de la lengua española. Madrid: Gredos, 1981.

RAVID, Dorit; MASHRAKI, Yael Epel. Prosodic reading, reading comprehension and morphological skills in Hebrew-speaking fourth graders. Journal of Research in Reading, v. 30, n. 2, p. 140-156, 2007. 
RECIO-PINEDA, Sara. Prosodia y comprensión lectora en Educación Primaria. 2017. 448f. Tese de doutoramento (Didáctica de la lengua y la literatura), Facultat d'Educació, Universitat de Barcelona, Barcelona, 2017.

SABATINI, John; WANG, Zuowei; O’ReILly, Tenaha. Relating Reading Comprehension to Oral Reading Performance in the NAEP Fourth-Grade Special Study of Oral Reading. Reading Research Quarterly, v. 54, n. 2, p. 253-271, 2019.

TsuI, Rachel Ka-Ying; Tong, Xiuli; Fung, LEO Shing-Chun. The role of prosodic reading in English reading comprehension among CantoneseEnglish bilingual children. Speech Prosody, 31 May - 3 Jun, p. 582-586, 2016.

WALCZYK, Jeffrey J.; GRIFFITH-Ross, Diana A. How important is reading skill fluency for comprehension? The Reading Teacher, v. 60, n. 6, p. 560569, 2007.

YILDIRIM, Kasim; RASINSKI, Timothy; KAYA, Dudu. Fluency and Comprehension of Expository Texts in Turkish Students in Grades Four through Eight. Education and Science, v. 42, n. 192, p.87-98, 2017.

Recibido en: 31/08/2019

Aceptado en: 14/02/2020

Title: Reading speed, prosody, and comprehension results

Título: Velocidade de leitura, prosódia e resultados de compreensão 